\title{
Local Electric Fields in Aqueous Electrolytes
}

\author{
Published as part of The Journal of Physical Chemistry virtual special issue "Dor Ben-Amotz Festschrift". \\ Chad I. Drexler, Olivia M. Cracchiolo, Ryan L. Myers, Halil I. Okur, Arnaldo L. Serrano,* \\ Steven A. Corcelli,* and Paul S. Cremer*
}

Cite This: J. Phys. Chem. B 2021, 125, 8484-8493

Read Online

ACCESS | Lلw Metrics \& More | 回 Article Recommendations | S1 Supporting Information

\begin{abstract}
Vibrational Stark shifts were explored in aqueous solutions of organic molecules with carbonyl- and nitrile-containing constituents. In many cases, the vibrational resonances from these moieties shifted toward lower frequency as salt was introduced into solution. This is in contrast to the blue-shift that would be expected based upon Onsager's reaction field theory. Salts containing well-hydrated cations like $\mathrm{Mg}^{2+}$ or $\mathrm{Li}^{+}$led to the most pronounced Stark shift for the carbonyl group, while poorly hydrated cations like $\mathrm{Cs}^{+}$had the greatest impact on nitriles. Moreover, salts containing $\mathrm{I}^{-}$gave rise to larger Stark shifts than those containing $\mathrm{Cl}^{-}$. Molecular dynamics simulations indicated that cations and anions both accumulate around the probe in an ion- and probe-dependent manner. An electric field was generated by the ion pair, which pointed from the cation to the anion through the vibrational chromophore. This resulted from solvent-shared binding of the ions to the probes, consistent with their positions in the Hofmeister series. The "anti-Onsager" Stark shifts occur in both vibrational spectroscopy and fluorescence measurements.
\end{abstract}

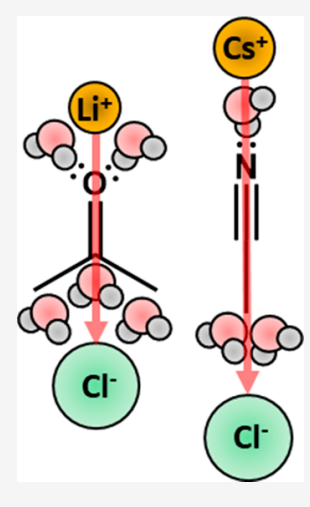

\section{INTRODUCTION}

The local response of a dielectric solvent to the presence of a polar solute is described by Onsager's reaction field theory. ${ }^{1}$ In this model, the dipole moment gives rise to polarization in the surrounding solvent that creates a local electric field aligned parallel to the dipole. Onsager's theory is a continuum model, which relates the field to the solvent polarity (eq 1$){ }^{2}$

$$
\vec{F}_{\mathrm{rxn}}=\frac{\vec{\mu}}{4 \pi \varepsilon_{0} a^{3}} \frac{2\left(\varepsilon_{\mathrm{r}}-1\right)\left(n^{2}+2\right)}{3\left(n^{2}+2 \varepsilon_{\mathrm{r}}\right)}
$$

$\vec{F}_{\text {rxn }}$ is the solvent reaction field, $\vec{\mu}$ is the dipole moment of the solute, $\varepsilon_{\mathrm{r}}$ is the relative static permittivity of the solvent $\left(\varepsilon_{\mathrm{r}}=\right.$ 78.54 for pure water at $25{ }^{\circ} \mathrm{C}$ ), $n$ is the refractive index of the solute, and $a$ is the radius of the cavity occupied by the solute. The local reaction field couples back to the solute, which gives rise to a Stark shift in the optical and vibrational spectra of polar chromophores. ${ }^{3-6}$ As such, modulating the solvent's polarity can lead to pronounced changes in the color of visible dyes through an effect known as optical solvatochromism. ${ }^{7-11}$ Moreover, vibrational solvatochromism has been observed in molecular probes with $\mathrm{C}=\mathrm{O}$ and $\mathrm{C} \equiv \mathrm{N}$ bonds. ${ }^{12-36}$ This Stark shift has been exploited to estimate electric fields in catalysis. $^{37-41}$

The use of molecular probes to measure local electric fields relies on preserving the bond force constant of the probe when the environment is changed. ${ }^{37-41}$ Indeed, a vibrational Stark shift is a dipole-field coupling phenomenon whereby the field interacts more strongly with the excited state than the ground state of an anharmonic oscillator due to the increase in the bond dipole. $^{33}$ This results in greater stabilization of the excited state, and the frequency is expected to red-shift with increasing solvent polarity. However, a change in anharmonicity of the probe can occur if the molecule participates in hydrogen bonding. ${ }^{36,42,43}$ For example, charge transfer from a lone pair on carbonyls or nitriles into antibonding orbitals on first shell water molecules can modify the force constant of the stretching modes. ${ }^{44}$ This complicates measurements of electric fields in water. Another potential complication involves the effects of through-space electric fields from ions in aqueous electrolytes. To date, only limited work has been performed to understand Stark shifts in the presence of salts.

Herein, we explore the reaction field of aqueous salt solutions by vibrational, two-dimensional infrared (2DIR) and florescence spectroscopies as well as by molecular dynamics (MD) simulations. A red-shift in the vibrational resonance of hydrated $\mathrm{C}=\mathrm{O}$ and $\mathrm{C} \equiv \mathrm{N}$ moieties was typically observed as a function of salt concentration between neat water and saturation conditions. This observation is at odds with Onsager's model because the bulk dielectric constant of liquid water decreases upon the addition of salt. As such, the field given by eq 1 is weakened, and a blue-shift should be

Received: April 11, 2021

Revised: July 5, 2021

Published: July 27, 2021

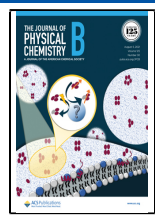




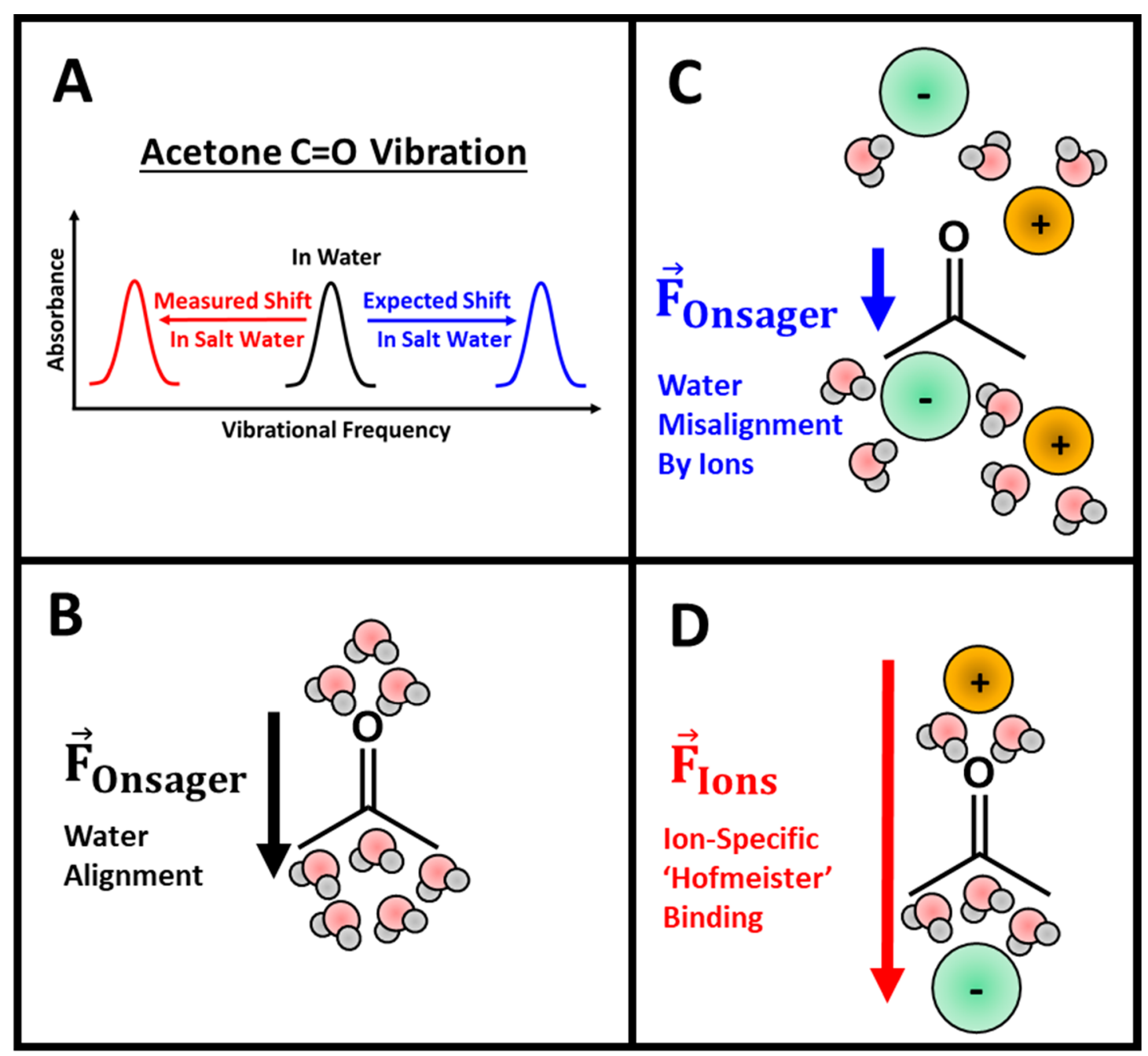

Figure 1. (A) Schematic illustration of the effect of salt on the $\mathrm{C}=\mathrm{O}$ stretch mode from acetone. (B) Dipole moment of acetone orders water around it to give rise to a local field. The black arrow depicts the initial field present for the black peak in (A) before salt is introduced. (C) Addition of salt gives rise to the formation of ionic hydration shells. Such water molecules are less available to be aligned by acetone than in pure water. This might be expected to result in a weakening of the field from water dipoles. The blue arrow corresponds to the field expected for the blue-shifted peak in (A). (D) Water-mediated interactions between the probe and the ions result in a net field which points from the cation to the anion. The field in this case is larger than in (B), as opposed to the field in (C), which is smaller than in (B).

expected instead (Figure 1A). Such “anti-Onsager” solvatochromism has previously been observed in salt solutions, although a molecular-level understanding of the local field remains elusive. ${ }^{4,46}$ Onsager's model is consistent with a molecular-level picture of how a molecular dipole, like a carbonyl group, would be expected to align water molecules to create a local field (Figure 1B). Of course, the alignment of the solvent will be disrupted by the introduction of ions, which would nominally lead to a blue-shift in the vibrational resonance (Figure 1C). The local field, however, is also dependent on the positioning of the ions, which can result in a red-shift instead (Figure 1D). As demonstrated herein, the ion effect depicted in Figure $1 \mathrm{D}$ is often dominant and is dependent on specific ion interactions with the chromophore. As such, the observed shifts follow a Hofmeister series that depends on the specific functional groups on the probe.

\section{MATERIALS AND METHODS}

Sample Preparation and Material Use. All samples were freshly prepared before use. The concentration of all carbonyl or nitrile probes was $0.1 \mathrm{M}$ unless stated otherwise. All carbonyl compounds and accompanying salts were dissolved in $\mathrm{D}_{2} \mathrm{O}$ to avoid overlap between the $\mathrm{H}_{2} \mathrm{O}$ bending mode and the carbonyl or amide I resonance. Reference solutions for linearIR measurements were prepared by adding salt to $\mathrm{D}_{2} \mathrm{O}$. Acetonitrile and the accompanying salts were dissolved in $\mathrm{H}_{2} \mathrm{O}$ to avoid overlap between the $\mathrm{D}_{2} \mathrm{O}$ stretch and $\mathrm{C} \equiv \mathrm{N}$ resonances. PRODAN was added to solution at a concentration of $\sim 1 \mu \mathrm{M}$.

All materials were used as received. $\mathrm{D}_{2} \mathrm{O}$ solvent $(99.8 \% \mathrm{D}$ atom) was purchased from Sigma-Aldrich (St. Louis, MO). All salts used for FTIR and Raman measurements were purchased from Sigma-Aldrich (St. Louis, MO). $\mathrm{H}_{2} \mathrm{O}(18.2 \mathrm{M} \Omega \cdot \mathrm{cm}$ ) was produced with a Barnstead Nanopure water system (Thermo Fischer).

ATR-FTIR Collection. All linear-IR spectra presented here were collected on a Nicolet Fourier transform (FT) IR spectrometer (Thermo Fischer Scientific, Billerica, MA) equipped with an attenuated total internal reflection (ATR) stage as described elsewhere. ${ }^{47}$ The intrinsic resolution of the detector was $2 \mathrm{~cm}^{-1}$. Each spectrum was averaged over 64 scans. Blackman-Harris and ATR corrections were performed. 


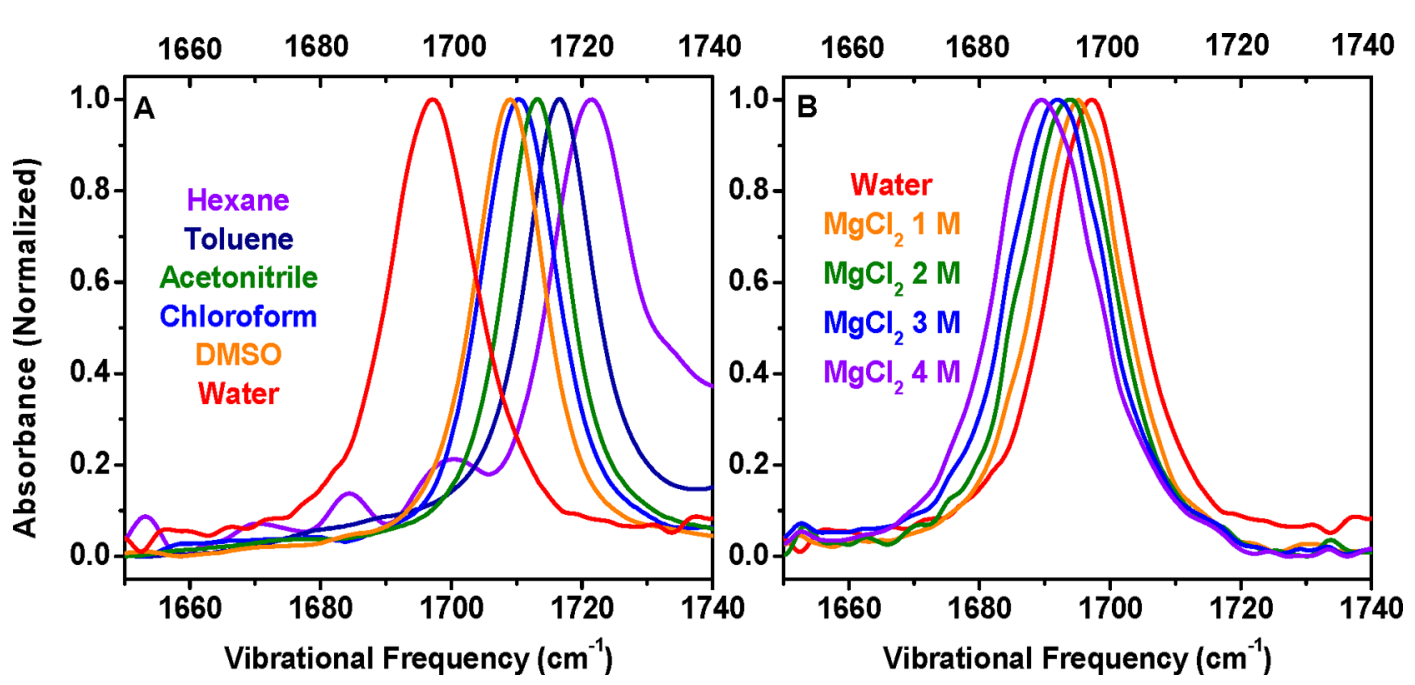

Figure 2. ATR-FTIR absorption spectra of the carbonyl stretch from acetone (A) in pure solvents and (B) in salt solutions. The acetone concentration was $0.1 \mathrm{M}$ in each case. Both spectral series are color-coded from large $\varepsilon_{\mathrm{r}}\left(\right.$ red; $\left.\mathrm{D}_{2} \mathrm{O}\right)$ to low $\varepsilon_{\mathrm{r}}(\text { violet; hexane or } 4 \mathrm{M} \mathrm{MgCl})_{2}$. All measurements were made at $25{ }^{\circ} \mathrm{C}$.

The ATR window was blanked to pure $\mathrm{D}_{2} \mathrm{O}$ for experiments conducted at $0 \mathrm{M}$ salt. A solution containing $0.1 \mathrm{M}$ of the carbonyl-containing compound was then placed on the ATR window. For spectra of $\mathrm{C}=\mathrm{O}$ vibrations in the presence of salt, the window was blanked to the corresponding $\mathrm{D}_{2} \mathrm{O}$ salt solution.

Raman Collection. Raman spectroscopy was used to measure samples containing acetonitrile. This was done because the nitrile stretch was highly Raman active, but the signal was weak in the IR. As such, to obtain sufficient signal from samples containing $0.1 \mathrm{M}$ acetonitrile, Raman was employed instead of IR. However, additional control experiments with $0.5 \mathrm{M}$ acetonitrile samples were performed by IR spectroscopy to check the Raman findings (see the Supporting Information for details). In fact, all conclusions from the Raman spectra were found to be the same in the IR. The Raman spectra were collected on a home-built spectrometer described previously. ${ }^{48}$ In brief, an ArKr ion laser (SpectraPhysics) was used to generate an excitation source at 514.5 $\mathrm{nm}$. The laser power used to illuminate the sample was $\sim 50$ $\mathrm{mW}$. The signal was sent to a spectrometer (Isoplane-SCT320; Princeton Instruments, Trenton, NJ) that spread the light onto a grating (1600 grooves $/ \mathrm{cm})$ before detection by a liquid nitrogen cooled $100 \times 1340 \mathrm{CCD}$ array camera (Pylon100BR; Princeton Instruments, Trenton, NJ). The signal was binned every $0.5 \mathrm{~cm}^{-1}$. Subpixel drifts were corrected with emission lines from a helium lamp employed as an internal reference.

2DIR Collection. All 2DIR spectra were collected on a home-built 2DIR spectrometer as described previously. ${ }^{49}$ Briefly, broadband mid-IR pulses are generated from an Astrella laser system (Coherent) outputting $3.5 \mathrm{~mJ}, 30 \mathrm{fs}, 800$ $\mathrm{nm}$ pulses at a repetition rate of $1 \mathrm{kHz}$. The beam is then directed to a TOPAS prime OPA (Light Conversion) and downconverted in a $\mathrm{AgGaS}_{2}$-based DFG. Pulses are then shaped by using an AOM (PhaseTech Spectroscopy) generating the mid-IR pulses required for the $2 \mathrm{D}$ experiment. The waiting time between the pump and probe pulses was fixed at 0 fs for all reported spectra. The probe beam is recollimated after the sample and redirected along with the reference beam to a home-built monochromator and then imaged onto a $128 \times 128$ mid-IR MCT array camera (Catalina, Teledyne).

Samples were prepared under dry air and sandwiched between two $\mathrm{CaF}_{2}$ windows. The laser table was purged under dry air to avoid interference with atmospheric water.

Molecular Dynamics (MD) Simulations. MD simulations were performed under ambient conditions by using the large-scale atomic/molecular massively parallel simulator (LAMMPS). ${ }^{50}$ The simulation box consisted of $1000 \mathrm{D}_{2} \mathrm{O}$ molecules modeled by using the SPC/E force field and two acetone solute molecules modeled with the general Amber force field (GAFF) with 72 calcium ions and 144 chloride ions added to represent a $4 \mathrm{M} \mathrm{CaCl}$ aqueous solution. The electronic continuum correction with ionic size refinement (ECCR2) force field was used to model the ions. ${ }^{51}$

After the preparation of the simulation box, equilibration began with 5000 minimization steps followed by a 5 ns simulation in the NPT ensemble at $300 \mathrm{~K}$ and 1 atm using a Nosé-Hoover thermostat. The simulation box was isotropically rescaled by using the last $4 \mathrm{~ns}$ of the NPT simulation to fix the size of the box consistent with 1 atm. Upon rescaling, the box was further equilibrated for $10 \mathrm{~ns}$ in the NVT ensemble and finally $1 \mathrm{~ns}$ in the NVE ensemble. The final simulations were run for $40 \mathrm{~ns}$ in the NVE ensemble with a $2 \mathrm{fs}$ time step.

Cylindrical Distribution Functions. The full MD trajectories were analyzed by using cylindrical distribution functions (CDFs). ${ }^{52}$ Similar to radial distribution functions, CDFs take a cylindrical slice centered around the atoms or bonds of interest, adding an additional dimension, and providing a more complete spatial representation. In this work, the CDFs are defined as $g(r, z)$ centered around the carbonyl bond, where the molecule is oriented along the $z$-axis and $r$ is the perpendicular distance to the $z$-axis.

\section{RESULTS}

Vibrational Solvatochromism of Acetone. In a first set of experiments, attenuated total internal reflection Fourier transform IR (ATR-FTIR) absorption spectra of the $\mathrm{C}=\mathrm{O}$ stretch mode of acetone were collected in a series of solvents (Figure 2A) as well as in aqueous $\mathrm{MgCl}_{2}$ solutions ranging in 
concentration from 0 to $4 \mathrm{M}$ (Figure 2B). The vibrational frequencies and the corresponding solvent $\varepsilon_{\mathrm{r}}$ values are provided in Table 1 .

Table 1. Vibrational Stark Shifts from the Carbonyl Stretch of Acetone ${ }^{a}$

\begin{tabular}{lcc}
\multicolumn{1}{c}{ solvent } & $\begin{array}{r}\text { dielectric constant } \\
\left(\varepsilon_{\mathrm{r}}\right)^{35,53,54}\end{array}$ & $\begin{array}{c}\text { vibrational frequency } \\
\left(\mathrm{cm}^{-1}\right)\end{array}$ \\
hexane & 1.89 & 1722 \\
toluene & 2.3 & 1716 \\
chloroform & 4.71 & 1710 \\
acetonitrile & 37.5 & 1713 \\
DMSO & 46.84 & 1709 \\
water $\left(\mathrm{D}_{2} \mathrm{O}\right)$ & 78.54 & 1697 \\
$1 \mathrm{M} \mathrm{MgCl}_{2}(\mathrm{aq})$ & 57.1 & 1695 \\
$2 \mathrm{M} \mathrm{MgCl}_{2}(\mathrm{aq})$ & 49.0 & 1693 \\
$3 \mathrm{M} \mathrm{MgCl}_{2}(\mathrm{aq})$ & 44.3 & 1692 \\
$4 \mathrm{M} \mathrm{MgCl}_{2}(\mathrm{aq})$ & 42.0 & 1690
\end{tabular}

${ }^{a}$ Both the solvent dielectric constant and the vibrational frequency of acetone are provided for each spectrum in Figure 2. A plot of the acetone resonance frequency values shown in this table against the Onsager model (eq 1) is provided in Figure S1.

In the gas phase, $\varepsilon_{\mathrm{r}}=1.00$, the carbonyl stretch of acetone is at $1731 \mathrm{~cm}^{-1}$. The peak red-shifts to $1722 \mathrm{~cm}^{-1}$ when dissolved in hexane $\left(\varepsilon_{\mathrm{r}}=1.89\right)$. Moreover, as can be seen in Figure $2 \mathrm{~A}$, it continuously red-shifts as the dielectric constant of the solvent increases, reaching a value of $1697 \mathrm{~cm}^{-1}$ in neat water where $\varepsilon_{\mathrm{r}}=78.54$. These red-shifts represent a classical vibrational Stark effect. Once in water, however, lowering the bulk dielectric constant by introducing $\mathrm{MgCl}_{2}$ to the solution led to a further decrease in the carbonyl stretch frequency. At 4 $\mathrm{M} \mathrm{MgCl}_{2}$, the frequency fell to $1690 \mathrm{~cm}^{-1}$. This result is curious because the bulk dielectric constant of $4 \mathrm{M} \mathrm{MgCl}_{2}\left(\varepsilon_{\mathrm{r}}\right.$ $=42)$ is lower than DMSO $\left(\varepsilon_{\mathrm{r}}=46.84\right)$. The origin of this saltinduced red-shift is explored below.

The continuous shift in the $\mathrm{C}=\mathrm{O}$ spectrum as a function of salt concentration is reminiscent of a Stark shift. However, it is also possible that the $\mathrm{C}=\mathrm{O}$ bond is weakened by changes in the hydrogen bonding between acetone and water caused by the ions. To distinguish between these two possibilities, the anharmonicity of the carbonyl resonance was measured with 2DIR spectroscopy (Figure 3). ${ }^{55}$ Each vibrational mode in a 2DIR spectrum appears as a pair of peaks along the probe axis. The red peak corresponds to the ground state bleach and stimulated emission ( $0-1$ transition), while the blue peak corresponds to the excited state absorption (1-2 transition) of the $\mathrm{C}=\mathrm{O}$ bond. In the absence of salt, the center frequency of the $0-1$ transition is $1690.0 \mathrm{~cm}^{-1}$, while that of the $1-2$ transition is $1673.8 \mathrm{~cm}^{-1}$ (Figures 3A,C) Thus, the anharmonic shift is $16.2 \pm 0.4 \mathrm{~cm}^{-1}$. Both resonances redshift upon adding $4 \mathrm{M} \mathrm{MgCl}_{2}$ to the solution (Figures 3B,D). Nevertheless, the anharmonic shift remains essentially unchanged at $16.0 \pm 0.1 \mathrm{~cm}^{-1}$. This suggests that the ion-
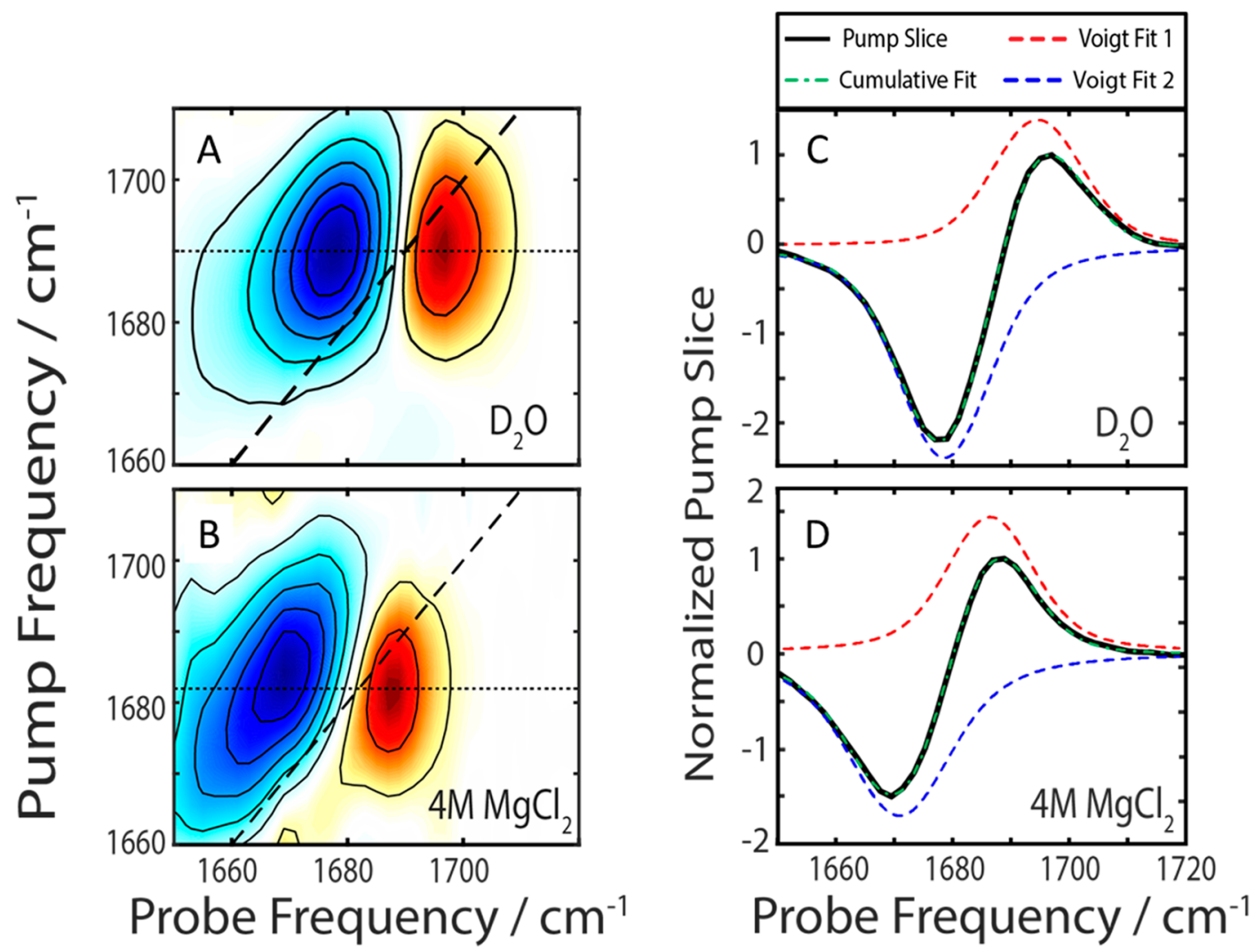

Figure 3. 2DIR spectra of acetone in (A) $\mathrm{D}_{2} \mathrm{O}$ and (B) $4 \mathrm{M} \mathrm{MgCl}_{2}$ in $\mathrm{D}_{2} \mathrm{O}$. Horizontal slices represented by the dotted lines in panels $\mathrm{A}$ and $\mathrm{B}$ demarcate the center frequency of the $0-1$ transition on the pump axis. These slices were taken at a pump frequency of $1690 \mathrm{~cm}^{-1}$ in $\mathrm{D}_{2} \mathrm{O}$ and $1685 \mathrm{~cm}^{-1}$ in $4 \mathrm{M} \mathrm{MgCl}_{2}$. On the other hand, the slanted dashed lines in (A) and (B) are diagonal slices from the 2D spectra where the pump and probe frequencies match one another. The horizontal slices from (A) and (B) are shown in (C) and (D), respectively, with a solid black curve which can be fit by using two Voigt functions. The peak frequencies extracted from the fits in (C) and (D) are for the $0-1$ transition (dashed red curves) and the 1-2 transitions (dashed blue curves). The overall fit to the two peaks is shown in green. 

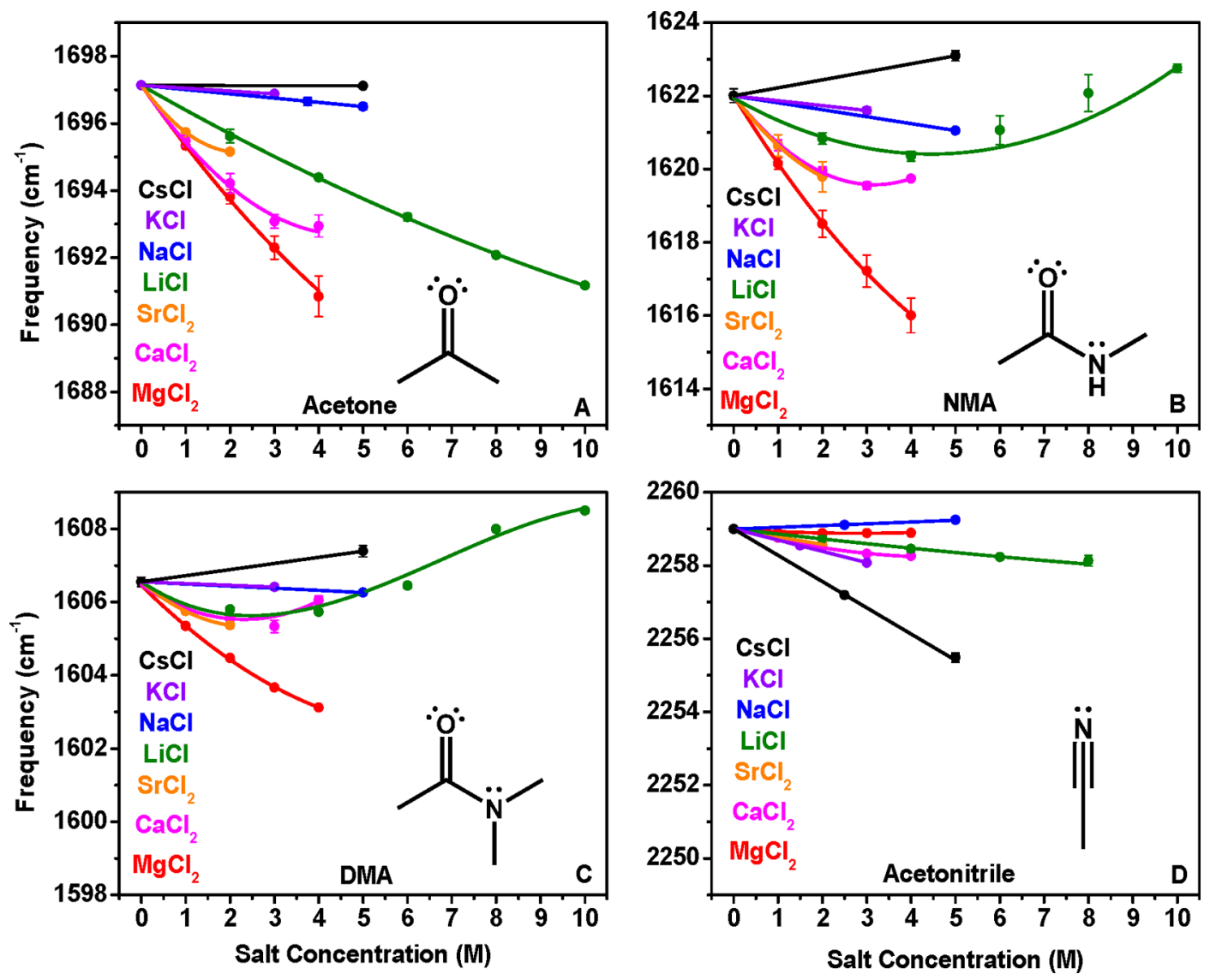

Figure 4. Solvatochromism of carbonyl and nitrile vibrations in salt solutions. The concentration of each probe was 0.1 M. Data are shown for (A) acetone, (B) N-methylacetamide (C) N,N-dimethylacetamide, and (D) acetonitrile. The colored curves are guides to the eye. Error bars correspond to averages of triplicate measurements. Infrared spectroscopy was used to obtain the data for the carbonyl probes while Raman was used for the nitrile probes (see the Supporting Information for details). Lewis structures of the individual probes are provided in each of the respective panels.

induced red-shift arises from a vibrational Stark effect as opposed to a modification in the probe force constant by analogy with previous work in pure solvents. ${ }^{55}$ This Stark shift, however, goes in the opposite direction of what would be predicted by Onsager's model.

Water-Mediated Interactions between Cations and Vibrational Probes. The experiments presented above suggest that the carbonyl resonance from acetone is redshifted by an electrostatic contribution from ions. Previous studies have, however, shown that the introduction of certain cations can lead to a new resonance that shows up as a blue shoulder approximately $20-25 \mathrm{~cm}^{-1}$ above the frequency of the main carbonyl resonance (i.e., in the case of amides). ${ }^{47,49,56}$ This happens when the cation contact pairs with the $\mathrm{C}=\mathrm{O}$ oxygen atom and dehydrates it (Figure S2). ${ }^{56}$ As such, $\mathrm{C}=\mathrm{O} \cdots$ $\mathrm{H}_{2} \mathrm{O}$ hydrogen bonds are displaced. ${ }^{57}$ By contrast, the continuous Stark shift in the hydrated bond resonance toward lower frequencies should arise from water-separated ions that act via a through space electric field. To better understand this phenomenon, we have tested a library of four probes as a function of salt concentration (Figure 4). Specifically, the behavior of acetone, $N$-methylacetamide (NMA), N,Ndimethylacetamide (DMA), and acetonitrile molecules was explored. The $y$-axis corresponds to the position of the center frequency of the hydrated carbonyl or nitrile mode upon the introduction of seven different chloride salts. The full spectrum for each probe with each of the salts is provided in the
Supporting Information (Figure S3). It should be noted that blue shoulders appear in some cases when salt is added.

As can be seen, changing the cation has a substantial and varied effect on the Stark shift. Chloride salts of well-hydrated metal cations gave rise to the most pronounced red-shift in the carbonyl frequency (Figure $4 \mathrm{~A}-\mathrm{C}$ ) as described by the following direct Hofmeister series at low salt concentrations:

$$
\mathrm{Mg}^{2+}>\mathrm{Ca}^{2+}>\mathrm{Sr}^{2+}>\mathrm{Li}^{+}>\mathrm{Na}^{+} \approx \mathrm{K}^{+}>\mathrm{Cs}^{+} \quad \text { (carbonyl series) }
$$

Curiously, a reverse Hofmeister series is observed for the $\mathrm{C} \equiv$ $\mathrm{N}$ stretching frequency (Figure 4D), which can be written as

$$
\mathrm{Cs}^{+}>\mathrm{K}^{+}>\mathrm{Ca}^{2+} \approx \mathrm{Sr}^{2+}>\mathrm{Li}^{+}>\mathrm{Na}^{+} \approx \mathrm{Mg}^{2+} \quad \text { (nitrile series) }
$$

The more pronounced red-shifts in the center frequencies of carbonyls by strongly hydrated cation chloride salts might suggest that the density of the electric field lines from ions dissolved in bulk solution governs the shift. However, a nearly opposite series is observed for nitriles, which speaks against such a mechanism. Instead, the Stark shift should be governed by the local organization of ions around the probes that results from their specific interactions with either the carbonyl or nitrile functional groups. For example, a weakly hydrated cation like $\mathrm{Cs}^{+}$would be expected to interact most readily with nitriles in accordance with hard-soft acid base (HSAB) theory. ${ }^{58}$ By contrast, well-hydrated cations, like $\mathrm{Mg}^{2+}$ and $\mathrm{Li}^{+}$, should interact more strongly with carbonyls. Significantly, this model suggests that specific ion effects govern the formation of 

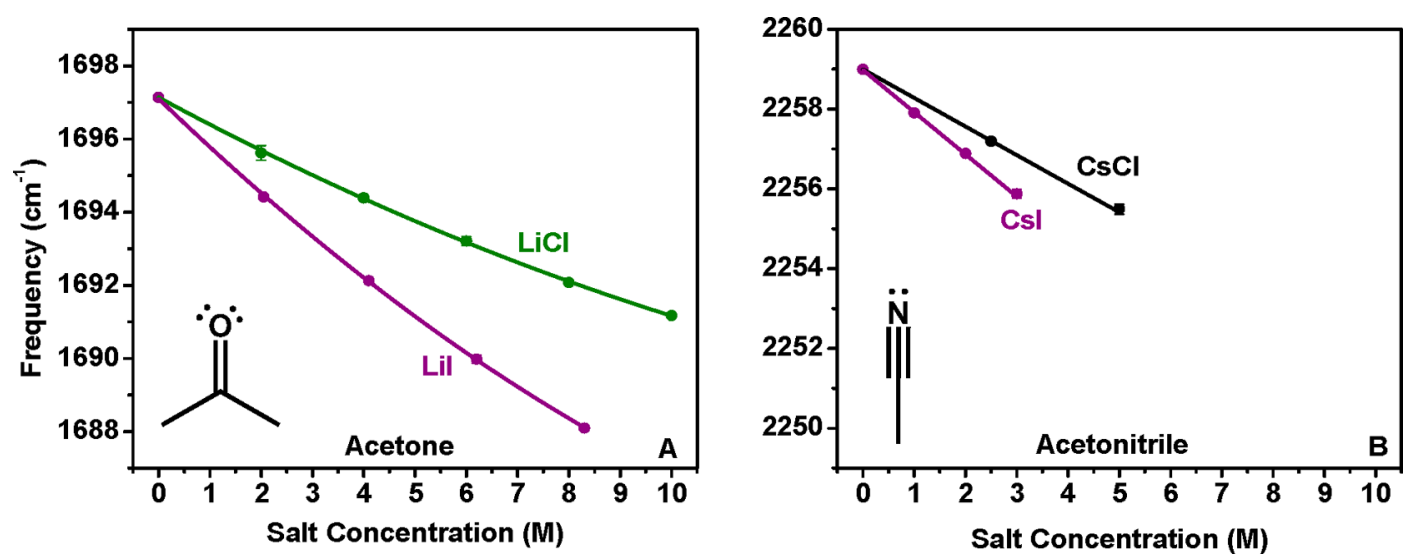

Figure 5. Influence of anions on salt-induced Stark shifts. Shifts in the vibrational frequency of (A) the hydrated C=O from acetone and (B) the hydrated $\mathrm{C} \equiv \mathrm{N}$ bond from acetonitrile are shown upon replacing $\mathrm{LiCl}$ with $\mathrm{LiI}$ and $\mathrm{CsCl}$ with CsI, respectively. The fits to the data are guides to the eye.
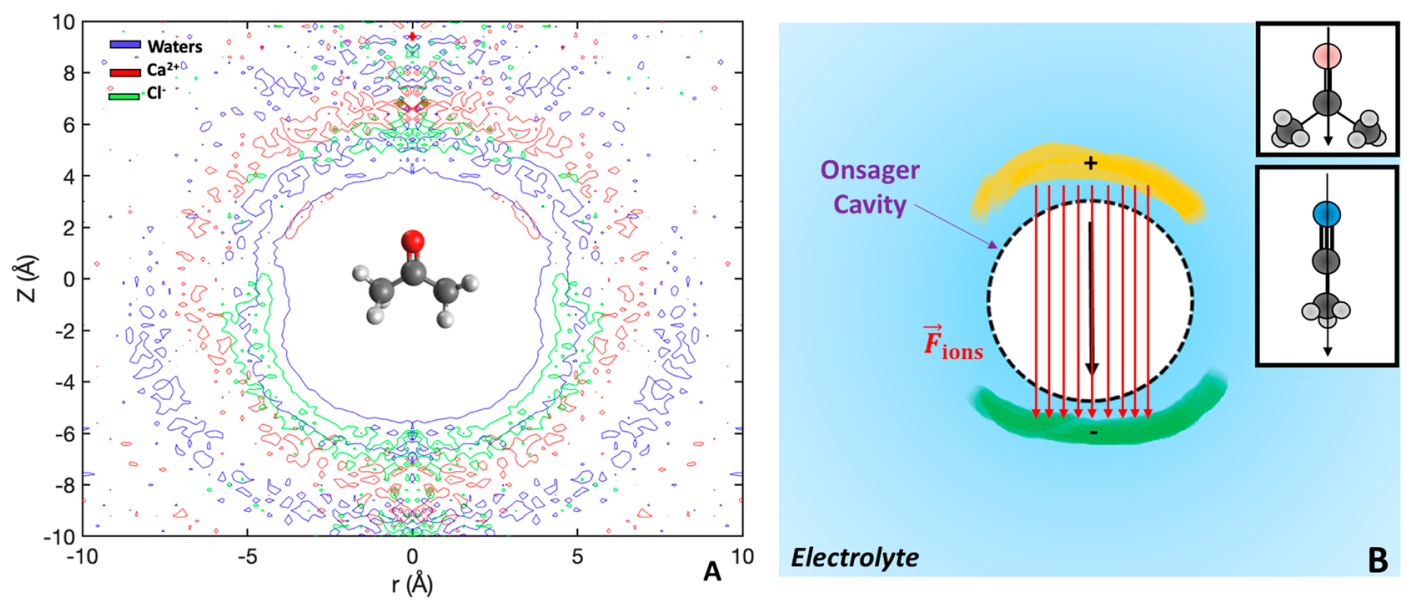

Figure 6. (A) Cylindrical distribution function produced from $\mathrm{MD}$ simulations of acetone and $4 \mathrm{M} \mathrm{CaCl}_{2}$ showing accumulation of Ca ${ }^{2+}$ (red), $\mathrm{Cl}^{-}$ (green), and water molecules (blue) around the dipole. (B) Schematic illustration of the proposed mechanism that gives rise to the red-going Stark shift found with numerous salts. The yellow region corresponds to cation accumulation and the green region to anion accumulation. The electric field lines shown in red, $\vec{F}_{\text {ions }}$, depict the net field from the accumulation of both ions. The mean molecular dipoles for acetone and acetonitrile are depicted in the upper and lower insets, respectively.

the local distribution of the ions around the probe. Once the distribution is established, however, the resulting Stark shifts can be thought of as resulting for point charges rather than from the specific nature of the ions.

Local Electric Field. Next, the counteranion was changed from $\mathrm{Cl}^{-}$to $\mathrm{I}^{-}$. This was done in conjunction with wellhydrated $\mathrm{Li}^{+}$cations in solutions containing acetone (Figure 5A) and with poorly hydrated $\mathrm{Cs}^{+}$cations in acetonitrile (Figure 5B). As can be seen, a substantially larger red-shift was observed upon the addition of LiI to solutions containing acetone compared to when $\mathrm{LiCl}$ was present. In fact, switching the anion nearly doubled the red-shift. Moreover, when CsI was introduced to solutions containing acetonitrile, the redshift was greater than when $\mathrm{CsCl}$ was added. These data are consistent with the notion that net salt accumulation around the probes was greater when $\mathrm{I}^{-}$was the anion. Indeed, $\mathrm{I}^{-}$is generally less excluded from the surface of uncharged organic molecules in water compared to $\mathrm{Cl}^{-59,60}$

To explore the accumulation of salts around the dipoles, classical MD simulations were performed in aqueous solutions containing acetone and $4 \mathrm{M} \mathrm{CaCl}_{2} \cdot \mathrm{Ca}^{2+}$ was used because the long water exchange times associated with hydrated $\mathrm{Mg}^{2+}$ made it impractical to employ. Cylindrical distribution functions were constructed from trajectory snapshots of the simulations (Figure 6A). In this approach, the transverse axis of the cylinder $(Z)$ was placed on the vertical axis and the radius of the cylinder $(r)$ was placed on the horizontal one. The system was centered around the carbonyl bond, which was oriented along the $Z$-axis. Introducing $4 \mathrm{M} \mathrm{CaCl}_{2}$ into these simulations resulted in a clear ordering of ions around the dipole of the solute. $\mathrm{Ca}^{2+}$ accumulated adjacent to the first hydration shell above the oxygen atom. A small fraction of $\mathrm{Cl}^{-}$ ions were located there as well, which was likely due to the formation of $\mathrm{CaCl}^{+}$ion pairs at $4 \mathrm{M}$. Significantly, however, the majority of $\mathrm{Cl}^{-}$sat underneath the dipole separated from acetone by a layer of water. This was clarified by plotting the normalized density of each ion (Figure S4), which revealed that most anions were located at negative $Z$ positions in Figure $6 \mathrm{~A}$, while most cations were found at positive $Z$ positions.

The results in Figure 6A support the model shown in Figure $6 \mathrm{~B}$, which is consistent with Stark shifts to the red upon the introduction of salt. Indeed, the "anti-Onsager" Stark shifts found in Figures 4 and 5 should arise from the double-ion bound construction that is depicted. When this ion structure is 
present around the organic species, the net local electric field from the ions, $\vec{F}_{\text {ions }}$, points from the cation to the anion. Indeed, by symmetry, $\vec{F}_{\text {ions }}$ will always possess a component aligned parallel to the mean molecular dipole. This should result in a universal red-shift as described by eq 2 .

$$
h c \Delta \bar{\nu}_{\text {salt }}=-\Delta \vec{\mu} \cdot \Delta \vec{F}
$$

Here, $\Delta \bar{\nu}_{\text {salt }}$ represents the shift in frequency of the probe upon the addition of salt to the solution. $\Delta \vec{\mu}$ is the Stark tuning rate defined to be the difference in the bond dipole between the ground and the first excited vibrational energy levels of the $\mathrm{C}=\mathrm{O}$ or $\mathrm{C} \equiv \mathrm{N}$ bonds (see the Supporting Information). For probes like acetone and acetonitrile, $\Delta \vec{\mu}$ should be well aligned with the mean molecular dipole. This idea is supported by the cylindrical distribution functions shown in Figure $S 4 . \Delta \vec{F}$ is the change in local field upon the addition of salt to solution. The change in field should be correlated to $\vec{F}_{\text {ions }}$ as depicted by the ion configuration in Figure 1D (see the Supporting Information). Moreover, the red-shift can be directly mapped to a density of local field lines (eq 2), which should increase as the concentration of double-ion bound dipoles increases.

\section{DISCUSSION AND CONCLUSIONS}

Herein, it was shown that the vibrational resonance of carbonyl and nitrile probes shows a Stark shift to the red upon introduction of salt to solution. This is in contrast to the blueshifted shoulder that has been previously reported for cation binding events in which the probe is dehydrated (e.g., $\mathrm{Ca}^{2+}$ interaction with NMA). ${ }^{49,56}$ The molecular origins of this redshifted Stark effect are discussed below.

Figure 4 reveals that interactions between cations and the probes occur in a way that is reminiscent of HSAB theory. This theory typically considers oxygen to be a hard ligand, while nitrogen is treated as relatively soft. ${ }^{61}$ As such, the interaction between $\mathrm{Li}^{+}$, a relatively hard cation, and the carbonyl oxygen atom is expected to be stronger than for other monovalent cations. Divalent cations are yet harder still and interact even better with the oxygen, which gives rise to an even greater redshift. Such findings are in agreement with a recent study that used acrylamide polymers. In that case, it was determined that well-hydrated cations locally accumulate around the amide oxygen from poly $(\mathrm{N}$-isopropylacrylamide $)$ in a water-mediated interaction in accordance with a direct Hofmeister series. ${ }^{62}$ This is indeed observed herein for acetone, NMA, and DMA. Moreover, a reverse Hofmeister series was found in the case of acetonitrile. Reversal of the cation series occurred because soft cations like $\mathrm{Cs}^{+}$interact more readily with the soft nitrogen atom than harder cations like $\mathrm{Li}^{+}$or $\mathrm{Mg}^{2+}$. In fact, wellhydrated cations hardly give rise to a Stark shift in acetonitrile. Such a finding is consistent with nearly negligible ion binding at the positions depicted in Figure 1D. Moreover, nitriles are known to display more complex frequency maps than carbonyls due to differences in how the nitrile vibration responds to hydrogen bonding at one position along the bond versus another. ${ }^{25}$ However, such complications do not likely govern the Stark shifts shown in Figure 4. Indeed, the reversal of the cation series in Figure 4D follows HSAB theory for both monovalent and divalent cation salts.

The data in Figure 5 reveal that the anion makes a crucial contribution to the local field. Specifically, $\mathrm{I}^{-}$is expected to be less depleted from the positive terminus of acetone and acetonitrile than $\mathrm{Cl}^{-}$. Previous investigations of Hofmeister anions have found that weakly hydrated $\mathrm{I}^{-}$anions interact with hydrophobic moieties on polymers more readily than $\mathrm{Cl}^{-} .63$ However, even $\mathrm{I}^{-}$has been shown to be depleted from the first hydration shell of small organic molecules such as alcohols. ${ }^{64}$ In the current experiments, both anions should therefore be depleted from the first hydration shell of the methyl groups, and the results in Figure $6 \mathrm{~A}$ are consistent with this notion. Moreover, since $\mathrm{I}^{-}$is less depleted, it allows a greater concentration of $\mathrm{Li}^{+}$to be present on the other side of the dipole. This increase in the local concentrations of both ions gives rise to a larger red-shift. As such, a direct Hofmeister series for anions is observed.

The local nature of $\vec{F}_{\text {ions }}$ does not rule out the presence of a standard Onsager effect from the bulk solution. Indeed, one can see evidence for this in the salt concentration dependence of the Stark shifts shown in Figure 4. For example, $\mathrm{LiCl}$ causes a red-shift of the NMA and DMA probe resonances at low salt concentrations, but this shift turns around at higher concentrations. This result is consistent with the idea that amide dipoles are populated with ions at low salt concentrations (Figure 1D) but become saturated as the concentration increases. Indeed, many of the probe resonances change nonlinearly at low salt concentration but appear to saturate like a Langmuir isotherm as the salt concentration is increased (Figures 4A-C). Simultaneously, the bulk dielectric constant of the solution continuously falls as salt is added, which should lead to a blue-shift in the probe frequency (Figure 1C). The turnaround point with NMA lies just beyond $4 \mathrm{M} \mathrm{LiCl}$ (Figure 4B), and the blue-shift becomes dominant at higher salt concentrations, in agreement with the classical Onsager model. A second example of the blue-shift effect can be seen from the interactions of $\mathrm{CsCl}$ with $\mathrm{NMA}$ and DMA (Figures 4B,C). In both these cases, the cation is sufficiently well depleted from the amide oxygen that essentially only the blue-shift is observed. In other words, the ions simply do not bind, and the situation reverts solely to the model depicted in Figure 1C. NMA and DMA are more sensitive to this effect compared to acetone (Figure 4A versus Figures $4 \mathrm{~B}$ and $4 \mathrm{C}$ ). This sensitivity difference is consistent with previous observations that $\Delta \vec{\mu}$ is larger for amide I resonances than it is for the $\mathrm{C}=\mathrm{O}$ stretch of ketones. ${ }^{39}$

Finally, we consider the influence of salt on optical solvatochromism. Specifically, the fluorescence spectrum of PRODAN has been measured in various solvents and salt solutions containing dilute $\mathrm{MgCl}_{2}$ (Figure S8). PRODAN is known to be very sensitive to changes in local polarity. ${ }^{65,66}$ When salt was introduced into solutions containing this dye, a red-shift was observed instead of the classical blue-shift. Such experiments are consistent with the idea that "anti-Onsager" effects impact fluorescence in additional to vibrational processes.

\section{ASSOCIATED CONTENT}

\section{(s) Supporting Information}

The Supporting Information is available free of charge at https://pubs.acs.org/doi/10.1021/acs.jpcb.1c03257.

Solvatochromic shifts and Onsager's model, dehydration of carbonyls by metal cations, linear vibrational spectra library, Stark shifts and covalent modifications monitored by Raman and ATR-FTIR, derivation of frequency-field relationships in salt solutions, optical 
solvatochromism of PRODAN in salt solutions, use of high salt concentrations in experimental data (PDF)

\section{AUTHOR INFORMATION}

\section{Corresponding Authors}

Arnaldo L. Serrano - Department of Chemistry and Biochemistry, University of Notre Dame, Notre Dame, Indiana 46556, United States; (1) orcid.org/0000-00027268-1306; Email: Arnaldo.serrano@nd.edu

Steven A. Corcelli - Department of Chemistry and Biochemistry, University of Notre Dame, Notre Dame, Indiana 46556, United States; 이이이.org/0000-00016451-4447; Email: scorcell@nd.edu

Paul S. Cremer - Department of Chemistry and Department of Biochemistry and Molecular Biology, The Pennsylvania State University, University Park, Pennsylvania 16802, United States; 이이.orcid.org/0000-0002-8524-0438; Email: psc11@psu.edu

\section{Authors}

Chad I. Drexler - Department of Chemistry, The Pennsylvania State University, University Park, Pennsylvania 16802, United States; 이이.org/0000-0002-9989-968X

Olivia M. Cracchiolo - Department of Chemistry and Biochemistry, University of Notre Dame, Notre Dame, Indiana 46556, United States

Ryan L. Myers - Department of Chemistry, The Pennsylvania State University, University Park, Pennsylvania 16802, United States

Halil I. Okur - Department of Chemistry and National Nanotechnology Research Center (UNAM), Bilkent University, 06800 Ankara, Turkey; $\odot$ orcid.org/0000-00022492-1168

Complete contact information is available at: https://pubs.acs.org/10.1021/acs.jpcb.1c03257

\section{Author Contributions}

C.I.D. and O.M.C. contributed equally to this work.

\section{Notes}

The authors declare no competing financial interest.

\section{ACKNOWLEDGMENTS}

P.S.C. thanks the National Science Foundation (CHE2004050) for support. C.I.D. thanks Bratoljub Milosavljevic for help with measurements taken using PRODAN and for insightful conversations. A.L.S. gratefully acknowledges support from the College of Science at the University of Notre Dame. S.A.C. is grateful for high-performance computing resources and support from the College of Science Center for Research Computing at the University of Notre Dame.

\section{REFERENCES}

(1) Onsager, L. Electric Moments of Molecules in Liquids. J. Am. Chem. Soc. 1936, 58, 1486-1493.

(2) Sorenson, S. A.; Patrow, J. G.; Dawlaty, J. M. Solvation Reaction Field at the Interface Measured by Vibrational Sum Frequency Generation Spectroscopy. J. Am. Chem. Soc. 2017, 139, 2369-2378.

(3) Slocum, J. D.; Webb, L. J. Nitrile Probes of Electric Field Agree with Independently Measured Fields in Green Fluorescent Protein Even in the Presence of Hydrogen Bonding. J. Am. Chem. Soc. 2016, 138, 6561-6570.
(4) Ma, J.; Pazos, I. M.; Zhang, W.; Culik, R. M.; Gai, F. Site-Specific Infrared Probes of Proteins. Annu. Rev. Phys. Chem. 2015, 66, 357377.

(5) Baiz, C. R.; Błasiak, B.; Bredenbeck, J.; Cho, M.; Choi, J.-H.; Corcelli, S. A.; Dijkstra, A. G.; Feng, C.-J.; Garrett-Roe, S.; Ge, N.-H.; et al. Vibrational Spectroscopic Map, Vibrational Spectroscopy, and Intermolecular Interaction. Chem. Rev. 2020, 120, 7152-7218.

(6) Dong, J.; Solntsev, K. M.; Tolbert, L. M. Solvatochromism of the Green Fluorescence Protein Chromophore and Its Derivatives. J. Am. Chem. Soc. 2006, 128, 12038-12039.

(7) Klymchenko, A. S. Solvatochromic and Fluorogenic Dyes as Environment-Sensitive Probes: Design and Biological Applications. Acc. Chem. Res. 2017, 50, 366-375.

(8) Klymchenko, A. S.; Mely, Y. In Progress in Molecular Biology and Translational Science; Morris, M. C., Ed.; Fluorescence-Based Biosensors; Academic Press: 2013; Vol. 113, Chapter 2, pp 35-58.

(9) Li, X.; Zhai, J.; Xie, X. The Hofmeister Anion Effect on Ionophore-Based Ion-Selective Nanospheres Containing Solvatochromic Dyes. Electroanalysis 2020, 32, 749-754.

(10) Carr, P. W. Solvatochromism, Linear Solvation Energy Relationships, and Chromatography. Microchem. J. 1993, 48, 4-28.

(11) Buncel, E.; Rajagopal, S. Solvatochromism and Solvent Polarity Scales. Acc. Chem. Res. 1990, 23, 226-231.

(12) Sarkar, S.; Maitra, A.; Banerjee, S.; Thoi, V. S.; Dawlaty, J. M. Electric Fields at Metal-Surfactant Interfaces: A Combined Vibrational Spectroscopy and Capacitance Study. J. Phys. Chem. B 2020, 124, 1311-1321.

(13) Voegtle, M. J.; Pal, T.; Pennathur, A. K.; Menachekanian, S.; Patrow, J. G.; Sarkar, S.; Cui, Q.; Dawlaty, J. M. Interfacial Polarization and Ionic Structure at the Ionic Liquid-Metal Interface Studied by Vibrational Spectroscopy and Molecular Dynamics Simulations. J. Phys. Chem. B 2021, 125, 2741-2753.

(14) Pennathur, A. K.; Voegtle, M. J.; Menachekanian, S.; Dawlaty, J. M. Strong Propensity of Ionic Liquids in Their Aqueous Solutions for an Organic-Modified Metal Surface. J. Phys. Chem. B 2020, 124, $7500-7507$.

(15) Sarkar, S.; Patrow, J. G.; Voegtle, M. J.; Pennathur, A. K.; Dawlaty, J. M. Electrodes as Polarizing Functional Groups: Correlation between Hammett Parameters and Electrochemical Polarization. J. Phys. Chem. C 2019, 123, 4926-4937.

(16) Shi, H.; Cai, Z.; Patrow, J.; Zhao, B.; Wang, Y.; Wang, Y.; Benderskii, A.; Dawlaty, J.; Cronin, S. B. Monitoring Local Electric Fields at Electrode Surfaces Using Surface Enhanced Raman Scattering-Based Stark-Shift Spectroscopy during Hydrogen Evolution Reactions. ACS Appl. Mater. Interfaces 2018, 10, 33678-33683.

(17) Patrow, J. G.; Wang, Y.; Dawlaty, J. M. Interfacial Lewis AcidBase Adduct Formation Probed by Vibrational Spectroscopy. J. Phys. Chem. Lett. 2018, 9, 3631-3638.

(18) Patrow, J. G.; Sorenson, S. A.; Dawlaty, J. M. Direct Spectroscopic Measurement of Interfacial Electric Fields near an Electrode under Polarizing or Current-Carrying Conditions. J. Phys. Chem. C 2017, 121, 11585-11592.

(19) Fafarman, A. T.; Webb, L. J.; Chuang, J. I.; Boxer, S. G. SiteSpecific Conversion of Cysteine Thiols into Thiocyanate Creates an IR Probe for Electric Fields in Proteins. J. Am. Chem. Soc. 2006, 128, 13356-13357.

(20) Stafford, A. J.; Ensign, D. L.; Webb, L. J. Vibrational Stark Effect Spectroscopy at the Interface of Ras and Rap1A Bound to the Ras Binding Domain of RalGDS Reveals an Electrostatic Mechanism for Protein-Protein Interaction. J. Phys. Chem. B 2010, 114, 1533115344 .

(21) Hu, W.; Webb, L. J. Direct Measurement of the Membrane Dipole Field in Bicelles Using Vibrational Stark Effect Spectroscopy. J. Phys. Chem. Lett. 2011, 2, 1925-1930.

(22) Stafford, A. J.; Walker, D. M.; Webb, L. J. Electrostatic Effects of Mutations of Ras Glutamine 61 Measured Using Vibrational Spectroscopy of a Thiocyanate Probe. Biochemistry 2012, 51, 27572767. 
(23) Shrestha, R.; Cardenas, A. E.; Elber, R.; Webb, L. J. Measurement of the Membrane Dipole Electric Field in DMPC Vesicles Using Vibrational Shifts of P-Cyanophenylalanine and Molecular Dynamics Simulations. J. Phys. Chem. B 2015, 119, 2869-2876.

(24) Błasiak, B.; Londergan, C. H.; Webb, L. J.; Cho, M. Vibrational Probes: From Small Molecule Solvatochromism Theory and Experiments to Applications in Complex Systems. Acc. Chem. Res. 2017, 50, 968-976.

(25) Oh, K. I.; Choi, J. H.; Lee, J. H.; Han, J. B.; Lee, H.; Cho, M. Nitrile and Thiocyanate IR Probes: Molecular Dynamics Simulation Studies. J. Chem. Phys. 2008, 128, 154504.

(26) Lee, H.; Choi, J. H.; Cho, M. Vibrational Solvatochromism and Electrochromism. II. Multipole Analysis. J. Chem. Phys. 2012, 137, 114307.

(27) DeCamp, M. F.; DeFlores, L.; McCracken, J. M.; Tokmakoff, A.; Kwac, K.; Cho, M. Amide I Vibrational Dynamics of NMethylacetamide in Polar Solvents: The Role of Electrostatic Interactions. J. Phys. Chem. B 2005, 109, 11016-11026.

(28) Kim, H.; Cho, M. Infrared Probes for Studying the Structure and Dynamics of Biomolecules. Chem. Rev. 2013, 113, 5817-5847.

(29) Kwac, K.; Cho, M. Machine Learning Approach for Describing Vibrational Solvatochromism. J. Chem. Phys. 2020, 152, 174101.

(30) Cho, M. Vibrational Solvatochromism and Electrochromism: Coarse-Grained Models and Their Relationships. J. Chem. Phys. 2009, 130, 094505.

(31) Ahmed, I. A.; Gai, F. Simple Method to Introduce an Ester Infrared Probe into Proteins. Protein Sci. 2017, 26, 375-381.

(32) Abaskharon, R. M.; Brown, S. P.; Zhang, W.; Chen, J.; Smith, A. B.; Gai, F. Isotope-Labeled Aspartate Sidechain as a Non-Perturbing Infrared Probe: Application to Investigate the Dynamics of a Carboxylate Buried inside a Protein. Chem. Phys. Lett. 2017, 683, 193-198.

(33) Fried, S. D.; Boxer, S. G. Measuring Electric Fields and Noncovalent Interactions Using the Vibrational Stark Effect. Acc. Chem. Res. 2015, 48, 998-1006.

(34) Fried, S. D.; Wang, L. P.; Boxer, S. G.; Ren, P.; Pande, V. S. Calculations of the Electric Fields in Liquid Solutions. J. Phys. Chem. B 2013, 117, 16236-16248.

(35) Fried, S. D.; Bagchi, S.; Boxer, S. G. Measuring Electrostatic Fields in Both Hydrogen-Bonding and Non-Hydrogen-Bonding Environments Using Carbonyl Vibrational Probes. J. Am. Chem. Soc. 2013, 135, 11181-11192.

(36) Levinson, N. M.; Fried, S. D.; Boxer, S. G. Solvent-Induced Infrared Frequency Shifts in Aromatic Nitriles Are Quantitatively Described by the Vibrational Stark Effect. J. Phys. Chem. B 2012, 116, 10470-10476.

(37) Fried, S. D.; Bagchi, S.; Boxer, S. G. Extreme Electric Fields Power Catalysis in the Active Site of Ketosteroid Isomerase. Science 2014, 346, 1510-1514.

(38) Wu, Y.; Boxer, S. G. A Critical Test of the Electrostatic Contribution to Catalysis with Noncanonical Amino Acids in Ketosteroid Isomerase. J. Am. Chem. Soc. 2016, 138, 11890-11895.

(39) Schneider, S. H.; Boxer, S. G. Vibrational Stark Effects of Carbonyl Probes Applied to Reinterpret IR and Raman Data for Enzyme Inhibitors in Terms of Electric Fields at the Active Site. J. Phys. Chem. B 2016, 120, 9672-9684.

(40) Fried, S. D.; Boxer, S. G. Electric Fields and Enzyme Catalysis. Annu. Rev. Biochem. 2017, 86, 387-415.

(41) Wu, Y.; Fried, S. D.; Boxer, S. G. A Preorganized Electric Field Leads to Minimal Geometrical Reorientation in the Catalytic Reaction of Ketosteroid Isomerase. J. Am. Chem. Soc. 2020, 142, 9993-9998.

(42) Choi, J. H.; Oh, K. I.; Lee, H.; Lee, C.; Cho, M. Nitrile and Thiocyanate IR Probes: Quantum Chemistry Calculation Studies and Multivariate Least-Square Fitting Analysis. J. Chem. Phys. 2008, 128, 134506.

(43) First, J. T.; Novelli, E. T.; Webb, L. J. Beyond pKa: Experiments and Simulations of Nitrile Vibrational Probes in Staphylococcal
Nuclease Show the Importance of Local Interactions. J. Phys. Chem. B 2020, 124, 3387-3399.

(44) Reed, A. E.; Curtiss, L. A.; Weinhold, F. Intermolecular Interactions from a Natural Bond Orbital, Donor-Acceptor Viewpoint. Chem. Rev. 1988, 88, 899-926.

(45) Mulder, W. H.; Párkányi, C. Theory of the Salt Effect on Solvatochromic Shifts and Its Potential Application to the Determination of Ground-State and Excited-State Dipole Moments. J. Phys. Chem. A 2002, 106, 11932-11937.

(46) Lewis, N. H. C.; Iscen, A.; Felts, A.; Dereka, B.; Schatz, G. C.; Tokmakoff, A. Vibrational Probe of Aqueous Electrolytes: The Field Is Not Enough. J. Phys. Chem. B 2020, 124, 7013-7026.

(47) Okur, H. I.; Kherb, J.; Cremer, P. S. Cations Bind Only Weakly to Amides in Aqueous Solutions. J. Am. Chem. Soc. 2013, 135, 50625067.

(48) Drexler, C. I.; Miller, T. C.; Rogers, B. A.; Li, Y. C.; Daly, C. A.; Yang, T.; Corcelli, S. A.; Cremer, P. S. Counter Cations Affect Transport in Aqueous Hydroxide Solutions with Ion Specificity. J. Am. Chem. Soc. 2019, 141, 6930-6936.

(49) Cracchiolo, O. M.; Geremia, D. K.; Corcelli, S. A.; Serrano, A. L. Hydrogen Bond Exchange and $\mathrm{Ca}^{2+}$ Binding of Aqueous $\mathrm{N}$ Methylacetamide Revealed by 2DIR Spectroscopy. J. Phys. Chem. B 2020, 124, 6947-6954.

(50) Plimpton, S. Fast Parallel Algorithms for Short-Range Molecular Dynamics. J. Comput. Phys. 1995, 117, 1-19.

(51) Martinek, T.; Duboué-Dijon, E.; Timr, Š.; Mason, P. E.; Baxová, K.; Fischer, H. E.; Schmidt, B.; Pluhařová, E.; Jungwirth, P. Calcium Ions in Aqueous Solutions: Accurate Force Field Description Aided by Ab Initio Molecular Dynamics and Neutron Scattering. J. Chem. Phys. 2018, 148, 222813.

(52) de Oliveira, D. M.; Bredt, A. J.; Miller, T. C.; Corcelli, S. A.; Ben-Amotz, D. Spectroscopic and Structural Characterization of Water-Shared Ion-Pairs in Aqueous Sodium and Lithium Hydroxide. J. Phys. Chem. B 2021, 125, 1439-1446.

(53) Harris, F. E.; O'Konski, C. T. Dielectric Properties of Aqueous Ionic Solutions at Microwave Frequencies. J. Phys. Chem. 1957, 61, 310-319.

(54) Mardolcar, U. V.; de Castro, C. A. N.; Santos, F. J. V. Dielectric Constant Measurements of Toluene and Benzene. Fluid Phase Equilib. 1992, 79, 255-264.

(55) Schneider, S. H.; Kratochvil, H. T.; Zanni, M. T.; Boxer, S. G. Solvent-Independent Anharmonicity for Carbonyl Oscillators. J. Phys. Chem. B 2017, 121, 2331-2338.

(56) Drexler, C. I.; Koehler, S. J.; Myers, R. L.; Lape, B. A.; Elacqua, E.; Cremer, P. S. Comment on "Arresting an Unusual Amide Tautomer Using Divalent Cations. J. Phys. Chem. B 2021, 125, 477478.

(57) Pluhařová, E.; Baer, M. D.; Mundy, C. J.; Schmidt, B.; Jungwirth, P. Aqueous Cation-Amide Binding: Free Energies and IR Spectral Signatures by Ab Initio Molecular Dynamics. J. Phys. Chem. Lett. 2014, 5, 2235-2240.

(58) Nalewajski, R. F. Electrostatic Effects in Interactions between Hard (Soft) Acids and Bases. J. Am. Chem. Soc. 1984, 106, 944-945. (59) Zhang, Y.; Furyk, S.; Bergbreiter, D. E.; Cremer, P. S. Specific Ion Effects on the Water Solubility of Macromolecules: PNIPAM and the Hofmeister Series. J. Am. Chem. Soc. 2005, 127, 14505-14510.

(60) Zhang, Y.; Cremer, P. S. Interactions between Macromolecules and Ions: The Hofmeister Series. Curr. Opin. Chem. Biol. 2006, 10, 658-663.

(61) Ho, T. L. Hard Soft Acids Bases (HSAB) Principle and Organic Chemistry. Chem. Rev. 1975, 75, 1-20.

(62) Bruce, E. E.; Okur, H. I.; Stegmaier, S.; Drexler, C. I.; Rogers, B. A.; van der Vegt, N. F. A.; Roke, S.; Cremer, P. S. Molecular Mechanism for the Interactions of Hofmeister Cations with Macromolecules in Aqueous Solution. J. Am. Chem. Soc. 2020, 142, 19094-19100.

(63) Okur, H. I.; Hladílková, J.; Rembert, K. B.; Cho, Y.; Heyda, J.; Dzubiella, J.; Cremer, P. S.; Jungwirth, P. Beyond the Hofmeister 
Series: Ion-Specific Effects on Proteins and Their Biological Functions. J. Phys. Chem. B 2017, 121, 1997-2014.

(64) Rankin, B. M.; Ben-Amotz, D. Expulsion of Ions from Hydrophobic Hydration Shells. J. Am. Chem. Soc. 2013, 135, 88188821.

(65) Vequi-Suplicy, C. C.; Coutinho, K.; Lamy, M. T. Optical Characterization of Prodan Aggregates in Water Medium. Phys. Chem. Chem. Phys. 2013, 15, 11800-11807.

(66) Samanta, A.; Fessenden, R. W. Excited State Dipole Moment of PRODAN as Determined from Transient Dielectric Loss Measurements. J. Phys. Chem. A 2000, 104, 8972-8975. 